\title{
Emittance optimization with dispersion free steering at LEP
}

\author{
R. Assmann, P. Raimondi,* G. Roy, and J. Wenninger ${ }^{\dagger}$ \\ CERN, 1211 Geneva 23, Switzerland \\ (Received 17 July 2000; published 11 December 2000)
}

\begin{abstract}
Residual vertical dispersion can be a significant performance limitation for the LEP collider because the associated vertical emittance increase reduces the luminosity of the machine. To make the search for orbits yielding small vertical emittances fast and deterministic, a simultaneous correction of the closed orbit and the residual dispersion was implemented at LEP. The principle of the correction and the resulting performance gains are discussed.
\end{abstract}

PACS numbers: 29.20.Dh, 29.27.Eg, 29.27.Fh

\section{INTRODUCTION}

In lepton storage rings, small beam sizes at collision points or in insertion devices are required for highest luminosity or brightness. In colliders, the luminosity $\mathcal{L}$ can be expressed as

$$
\mathcal{L}=\frac{k_{b} I_{b}^{2}}{4 \pi e^{2} f_{\text {rev }} \sigma_{x}^{*} \sigma_{y}^{*}},
$$

with $k_{b}$ the number of bunches per beam, $I_{b}$ the current per bunch, and $f_{\text {rev }}$ the revolution frequency. In the absence of local coupling, the horizontal and vertical beam sizes at the collision points $\sigma_{x}^{*}$ and $\sigma_{y}^{*}$ are given by $(u=x, y)$

$$
\left(\sigma_{u}^{*}\right)^{2}=\left(D_{u}^{*} \sigma_{\delta}\right)^{2}+\beta_{u}^{*} \varepsilon_{u}
$$

where $D_{u}^{*}$ is the dispersion, $\sigma_{\delta}$ is the relative energy spread, $\beta_{u}^{*}$ is the betatron function, and $\varepsilon_{u}$ is the beam emittance. In the presence of weak local coupling, the beam size receives an additional contribution proportional to the emittance of the orthogonal plane and to the coupling factor. The number of bunches, the betatron functions $\beta_{x}^{*}$ and $\beta_{y}^{*}$, and the horizontal emittance $\varepsilon_{x}$ are given by the machine and optics design. Optimization of the luminosity with those parameters is possible but limited by other constraints (e.g., the dynamic aperture, momentum aperture, ...). For example, the horizontal beam emittance can be optimized by a proper choice of the machine optics, a higher phase advance per cell yielding a smaller natural horizontal emittance. Dynamic aperture requirements may, however, limit the increase of phase advance.

The vertical emittance $\varepsilon_{y}$ is a powerful parameter for luminosity optimization. Neglecting beam-beam effects, it can be written approximately as

$$
\varepsilon_{y} \simeq \varepsilon_{y 0}+\kappa \varepsilon_{x}+r E^{2}\left(D_{y}^{\mathrm{rms}}\right)^{2},
$$

with $E$ the beam energy and $r$ a numerical coefficient. For an ideal planar storage ring the vertical rms dispersion

\footnotetext{
*Permanent address: SLAC, P.O. Box 4349, Stanford, CA 94309.

†Corresponding author.
}

$D_{y}^{\mathrm{rms}}$ and the global coupling $\kappa$ vanish, and the emittance is given by $\varepsilon_{y 0}$, the limit from quantum excitation due to the finite emission angle of synchrotron radiation photons. In general, $\varepsilon_{y 0}$ is many orders of magnitude smaller than practically achievable emittances. Residual coupling between the planes and a nonzero vertical dispersion, both due to unavoidable machine imperfections, lead to a finite vertical beam emittance. The global coupling parameter $\kappa$ is a function of $\Delta_{Q}$, the distance of the tunes to the difference coupling resonance, and can be expressed to a good approximation by

$$
\kappa=\frac{\Delta_{g}^{2}}{\Delta_{g}^{2}+2 \Delta_{Q}^{2}} .
$$

Here $\Delta_{g}$ is the width of the stop band around the coupling resonance where the horizontal and vertical tunes become "undefined" due to the orientation of the normal modes axes $[1,2]$. The stop band width or "closest tune approach" can be measured by sweeping the betatron tunes across each other.

We note that optimization of the vertical emittance is not critical for a machine operated well above the beambeam limit where it is determined by the strength of the beam-beam interaction. This is, for example, the case of LEP when it is operated at beam energy of $45 \mathrm{GeV}$.

\section{LEP operation at high energy}

The Large Electron Positron collider (LEP) is a $26.7 \mathrm{~km}$ circumference storage ring [2]. The two beams circulate in the same vacuum chamber and collide at four interaction points. Each beam consists of four equidistant bunches. The 500 beam position monitors installed in LEP measure the beam position in both planes. 261 horizontal and 312 vertical orbit correctors are available for orbit steering. At LEP, the best performances were traditionally obtained with the help of so-called golden orbits [3]. Such orbits were found empirically with global and local orbit corrections and with knobs generating specific patterns of dispersion waves over the ring. By continuously reusing and improving the corrector settings and steering of the 
golden orbits, peak performances are achieved by "natural" selection on the time scale of a few weeks to a few months. Choosing as a target for the orbit correction the flattest possible orbit does not give good results, even for a well aligned machine and for beam position monitor offsets smaller than $0.1 \mathrm{~mm}$.

After $7 \mathrm{yr}$ of operation at beam energies around $45 \mathrm{GeV}$, LEP started running above $80 \mathrm{GeV}$ in 1996. At those high energies the beam-beam limit is increased significantly due to the stronger radiation damping, and beam-beam tune shifts above 0.08 have been observed $[2,4]$. The beambeam limit is not reached even at the highest bunch currents. The strong increase of the horizontal emittance with energy, $\varepsilon_{x} \propto E^{2}$, is compensated by shifting the horizontal damping partition number from 1.0 to 1.6 and by increasing the horizontal phase advance per arc cell from $90^{\circ}$ to $102^{\circ}$. Though some beam-beam blowup is observed, the vertical emittance is mainly given by the beam tuning.

Coupling is generated at LEP by misaligned machine elements and by the four large experimental solenoids. At high energy the contribution of the latter becomes weaker since $\kappa_{\text {sol }} \propto 1 / E$. Imperfections of the solenoid coupling corrections are therefore less critical, but they may still contribute to local coupling around the collision points. Four pairs of skew quadrupoles around each of the four collision points are used for coupling correction.

When all solenoids and skew quadrupoles are switched off, the measured stop band width is $\Delta_{g} \approx 0.02$. It is mainly generated by rolled quadrupoles and vertical orbit offsets in the sextupoles. For a typical vertical closed orbit rms of $0.6 \mathrm{~mm}$, the orbit offsets in the sextupoles contribute to the total coupling with $\Delta_{g} \approx 0.006$. At a beam energy of $100 \mathrm{GeV}$ the stop band produced by the four solenoids is $\Delta_{g} \simeq 0.019$. After correction of the coupling, the width of the stop band is reduced to $\Delta_{g} \leq 0.002$. The correction is first performed with the solenoids off to compensate the machine coupling and in a second step with the solenoids switched on. For $\Delta_{g} \leq 0.002$, the contribution of coupling to the vertical emittance is negligible for the operating betatron tunes of $Q_{x}=98.35$ and $Q_{y}=96.18$; see Fig. 1 . The coupling correction is regularly checked to track variations from orbit changes.

At high energy the vertical rms dispersion becomes the main ingredient to the vertical beam emittance and beam size at the collision points because of its strong dependence on energy; see Eq. (3) [5]. To improve the LEP performance, work on dispersion correction was started in 1998. Both empirical optimization with dispersion bumps and a deterministic correction algorithm were studied. The best results were achieved with a deterministic orbit and dispersion correction scheme developed at SLAC, the so-called dispersion free steering (DFS) [6-9]. The dispersion free steering scheme implemented for LEP is described, and the impact on the machine performances is presented.

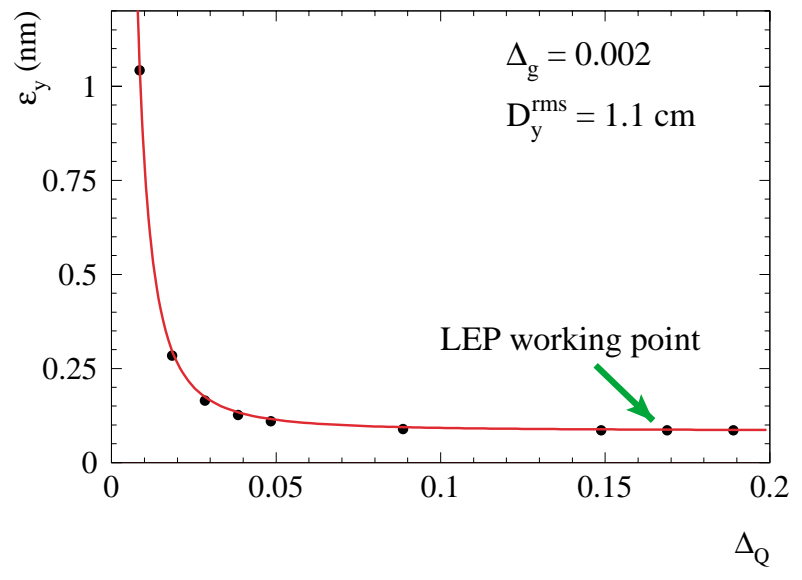

FIG. 1. (Color) Simulated dependence of the vertical emittance $\varepsilon_{y}$ for LEP as a function of the distance $\Delta_{Q}$ of the tunes from the difference coupling resonance. The beam energy is $100 \mathrm{GeV}$ and the horizontal emittance is $\varepsilon_{x}=44(\mathrm{~nm})$. The LEP working point corresponds to $\Delta_{Q}=0.17$ with horizontal tune $Q_{x}=98.35$ and vertical tune $Q_{y}=96.18$.

\section{DISPERSION FREE STEERING PRINCIPLE AND FORMALISM}

The principle of dispersion free steering consists of a simultaneous correction of the orbit and the dispersion using one of the standard orbit correction algorithms. This guarantees that the beam orbit is flat while at the same time minimizing the residual dispersion.

In most machines the beam position is measured with a set of $N$ beam position monitors (BPMs) which are distributed over the ring. The orbit is corrected with a set of $M$ dipole magnets (correctors). The beam position at the BPMs can be represented by a vector $\vec{u}$

$$
\vec{u}=\left(\begin{array}{c}
u_{1} \\
u_{2} \\
\cdots \\
u_{N}
\end{array}\right),
$$

and the corrector strengths (kicks) by a vector $\vec{\theta}$

$$
\vec{\theta}=\left(\begin{array}{c}
\theta_{1} \\
\theta_{2} \\
\ldots \\
\theta_{M}
\end{array}\right) .
$$

A response matrix $\mathbf{A}$ (dimension $N \times M$ ) is used to describe the relation between corrector kicks and beam position changes at the monitors. The element $A_{i j}$ of the response matrix corresponds to the orbit shift at the $i$ th monitor due to a unit kick from the $j$ th corrector.

The task of the orbit correction is to find a set of corrector kicks $\theta$ that satisfy the following relation:

$$
\vec{u}+\mathbf{A} \vec{\theta}=0 .
$$

In general, the number of BPMs $(N)$ and the number of correctors $(M)$ are not identical and Eq. (7) is either over 
$(N>M)$ or under $(N<M)$ constrained. In the former and most frequent case, Eq. (7) cannot be solved exactly. Instead, an approximate solution must be found, and commonly used least square algorithms minimize the quadratic residual

$$
S=\|\vec{u}+\mathbf{A} \vec{\theta}\|^{2} .
$$

Dispersion free steering is based on the extension of Eq. (7) to include the dispersion at the BPMs. The extended linear system is

$$
\left(\begin{array}{c}
(1-\alpha) \vec{u} \\
\alpha \vec{D}_{u}
\end{array}\right)+\left(\begin{array}{c}
(1-\alpha) \mathbf{A} \\
\alpha \mathbf{B}
\end{array}\right) \vec{\theta}=0
$$

where vector $\vec{D}_{u}$ (dimension $N$ ) represents the dispersion at the BPMs. B is the $N \times M$ dispersion response matrix, its elements $B_{i j}$ giving the dispersion change at the $i$ th monitor due to a unit kick from the $j$ th corrector. The weight factor $\alpha$ is used to shift from a pure orbit ( $\alpha=$ $0)$ to a pure dispersion correction $(\alpha=1)$. In general, the optimum closed orbit and dispersion rms are not of the same magnitude and $\alpha$ must be adjusted for a given machine. $\alpha$ can, in principle, be evaluated from the BPM accuracy and resolution. Applied to Eq. (9), a least square algorithm will minimize

$$
S=(1-\alpha)^{2}\|\vec{u}+\mathbf{A} \vec{\theta}\|^{2}+\alpha^{2}\left\|\vec{D}_{u}+\mathbf{B} \vec{\theta}\right\|^{2} .
$$

Singular response matrices are a well-known problem of orbit corrections. The singularities are related to redundant correctors, i.e., areas of the machine where the sampling of the orbit is insufficient. Such situations yield numerically unstable solutions where large kicks are associated to minor changes in the orbit. A standard cure consists in disabling a subset of correctors and removing the corresponding lines from the linear systems of Eqs. (7) and (9). Regularization can also be obtained by extending Eq. (9) to constrain the size of the kicks,

$$
\left(\begin{array}{c}
(1-\alpha) \vec{u} \\
\alpha \vec{D}_{u} \\
\overrightarrow{0}^{\prime}
\end{array}\right)+\left(\begin{array}{c}
(1-\alpha) \mathbf{A} \\
\alpha \mathbf{B} \\
\beta \mathbf{I}
\end{array}\right) \vec{\theta}=0 .
$$

Here $\overrightarrow{0}$ is a null vector of dimension $M$, I is a unit matrix of dimension $M \times M$, and $\beta$ is a kick weight. The quadratic residual now contains the rms strength of the corrector kicks,

$$
\begin{aligned}
S= & (1-\alpha)^{2}\|\vec{u}+\mathbf{A} \theta\|^{2}+\alpha^{2}\left\|\vec{D}_{u}+\mathbf{B} \vec{\theta}\right\|^{2} \\
& +\beta^{2}\|\vec{\theta}\|^{2},
\end{aligned}
$$

and large kicks are suppressed since they receive a penalty which can be adjusted with $\beta$.

Various other constraints can be added to the linear system to be solved, for example, to maintain a constant orbit length or to stabilize the beam at given locations in the ring. Adequate weight factors can be used to control the importance of such constraints. It is also possible to correct the machine coupling using a similar scheme. The orbit coupling of horizontal corrector kicks into the vertical plane is then minimized using skew quadrupoles as correcting elements [10]. To simplify the expressions in the following sections, vector $\vec{d}$ and matrix $\mathbf{T}$ are defined as

$$
\vec{d}=\left(\begin{array}{c}
(1-\alpha) \vec{u} \\
\alpha \vec{D}_{u} \\
\overrightarrow{0}
\end{array}\right), \quad \mathbf{T}=\left(\begin{array}{c}
(1-\alpha) \mathbf{A} \\
\alpha \mathbf{B} \\
\beta \mathbf{I}
\end{array}\right),
$$

with

$$
\vec{d}+\mathbf{T} \vec{\theta}=0 .
$$

\section{A. Singular value decomposition (SVD) and orbit eigenvectors}

Dispersion free steering is particularly interesting in conjunction with the singular value decomposition (SVD) algorithm [11,12], because it allows a simultaneous limitation of the corrector kick strength. The SVD algorithm is a powerful tool to handle singular systems and to solve them in the least square sense. For $M \geq N$ the singular value decomposition of matrix $\mathbf{T}$ has the form

$$
\mathbf{T}=\mathbf{U W V}^{\mathbf{t}}=\mathbf{U}\left(\begin{array}{cccc}
w_{1} & 0 & \cdots & 0 \\
0 & w_{2} & & \\
\cdots & & \cdots & 0 \\
0 & \cdots & 0 & w_{M}
\end{array}\right) \mathbf{V}^{\mathbf{t}},
$$

where $\mathbf{W}$ is a diagonal $M \times M$ matrix with non-negative diagonal elements and $\mathbf{V}^{\mathfrak{t}}$ is the transpose of the $M \times M$ orthogonal matrix $\mathbf{V}$,

$$
\mathbf{V V}^{\mathbf{t}}=\mathbf{V}^{\mathbf{t}} \mathbf{V}=\mathbf{I}
$$

while $\mathbf{U}$ is an $N \times M$ column-orthogonal matrix

$$
\mathbf{U}^{\mathbf{t}} \mathbf{U}=\mathbf{I} .
$$

The vector $\vec{\vartheta}^{(i)}$, corresponding to the $i$ th column of matrix $\mathbf{V}$,

$$
\vec{\vartheta}^{(i)}=\left(\begin{array}{c}
V_{1 i} \\
V_{2 i} \\
\cdots \\
V_{M i}
\end{array}\right)
$$

is an eigenvector with eigenvalue $w_{i}^{2} \geq 0$ of the $M \times M$ symmetric matrix $\mathbf{T}^{\mathbf{t}} \mathbf{T}[12,13]$,

$$
\mathbf{T}^{\mathbf{t}} \mathbf{T} \vec{\vartheta}^{(i)}=w_{i}^{2} \vec{\vartheta}^{(i)}
$$

It follows from Eq. (16) that the $M$ vectors $\vec{\vartheta}^{(i)}$ form an orthonormal base of the corrector space since

$$
\left(\vec{\vartheta}^{(i)} \cdot \vec{\vartheta}^{(j)}\right)=\vec{\vartheta}^{(i) \mathbf{t}} \vec{\vartheta}^{(j)}=\delta_{i j} .
$$




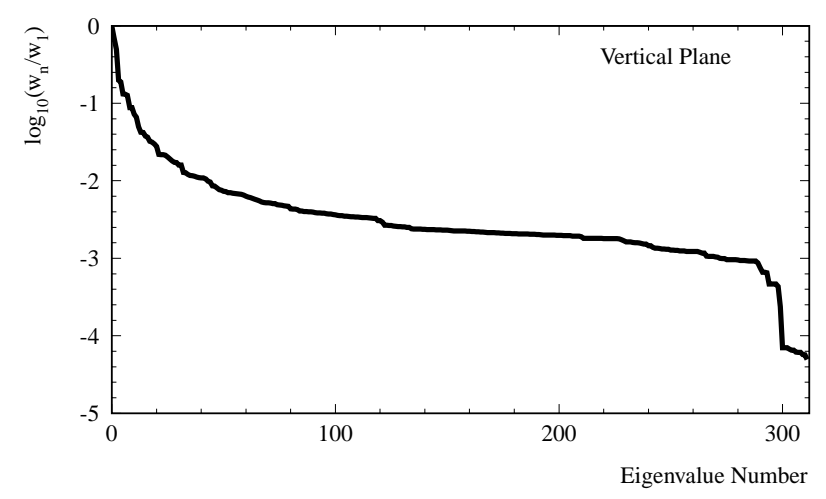

FIG. 2. Orbit eigenvalue spectrum for LEP in the vertical plane with $\alpha=0.2$ and $\beta=0$. The last four eigenvalues correspond to singular solutions in the low-beta sections around the interaction points.

Each eigenvector $\vec{\vartheta}^{(i)}$ is a linear combination of the usual physical correctors. The orbit and dispersion response $\overrightarrow{\boldsymbol{v}}^{(i)}$ associated to the kick eigenvector $\vec{\vartheta}^{(i)}$

$$
\overrightarrow{\boldsymbol{v}}^{(i)}=\mathbf{T} \vec{\vartheta}^{(i)}=\mathbf{U W V} \vec{\vartheta}^{(i)}=w_{i}\left(\begin{array}{c}
U_{1 i} \\
U_{2 i} \\
\ldots \\
U_{N i}
\end{array}\right)
$$

corresponds (modulo a factor $w_{i}$ ) to the $i$ th column of matrix $\mathbf{U}$. The $M$ vectors $\overrightarrow{\boldsymbol{v}}^{(i)}$ are orthogonal but not normalized,

$$
\left(\overrightarrow{\boldsymbol{v}}^{(i)} \cdot \overrightarrow{\boldsymbol{v}}^{(j)}\right)=\overrightarrow{\boldsymbol{v}}^{(i) \mathbf{t}} \overrightarrow{\boldsymbol{v}}^{(j)}=\overrightarrow{\boldsymbol{\vartheta}}^{(i) \mathbf{t}} \mathbf{T}^{\mathbf{t}} \mathbf{T} \overrightarrow{\boldsymbol{\vartheta}}^{(j)}=w_{i}^{2} \delta_{i j} .
$$

The eigenvalues (or weights) $w_{i}$ are a quantitative measure of the orbit and dispersion response to a given $\vec{\vartheta}^{(i)}$, since $\left(\vec{v}^{(i)} \cdot \vec{v}^{(i)}\right)^{1 / 2}$ is proportional to the orbit and dispersion rms associated to $\vec{v}^{(i)}$. The higher the eigenvalue, the
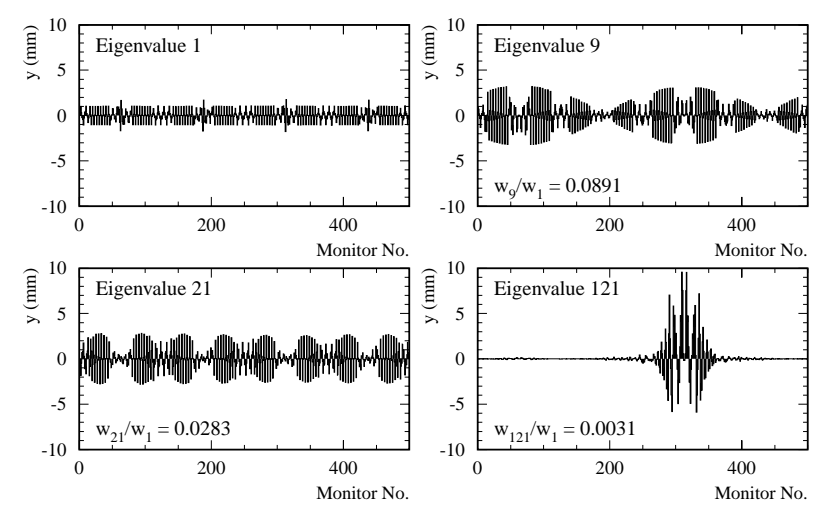

FIG. 3. Example of normalized orbit eigenvectors $\left(w_{1} \overrightarrow{\boldsymbol{v}}^{(i)} / w_{i}\right)$ for the vertical plane at LEP with $\alpha=0.2$ and $\beta=0$. Only the orbit component of the eigenvector is shown here. The harmonic content of the first eigenvectors reflects the machine symmetries. The main harmonics are $Q$ (the machine tune) for eigenvector $1, Q \pm 1$ for eigenvector 9 , and $Q \pm 4$ for eigenvector 21 . The eigenvectors associated to small eigenvalues (bottom right) often correspond to long "bumps" over some section of the machine.
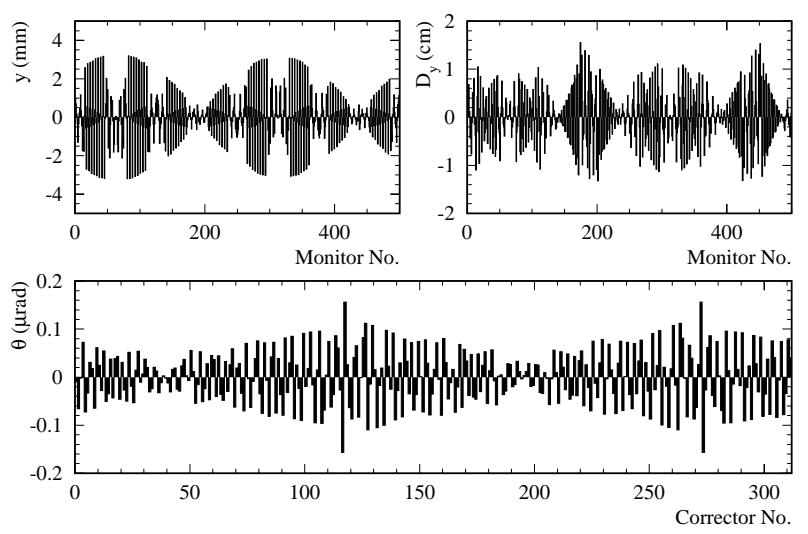

FIG. 4. Normalized orbit $\left(w_{1} \overrightarrow{\boldsymbol{v}}^{(9)} / w_{9}\right)$ and kick $\left(\overrightarrow{\boldsymbol{\vartheta}}^{(9)}\right)$ eigenvector for eigenvalue number 9 (see also Fig. 3). The orbit (top left) and dispersion (top right) components of $\vec{v}^{(9)}$ are shown together with the corrector setting corresponding to $\vec{\vartheta}^{(9)}$ (bottom).

larger the response. Very small eigenvalues correspond to singular solutions where combinations of correctors lead to essentially no response on the measured orbit or dispersion.

Examples of eigenvalue spectra and eigenvectors for LEP are shown in Figs. 2-4. By default, the eigenvalues and eigenvectors are always ordered by decreasing eigenvalue. The eigenvectors associated to the largest eigenvalues correspond to orbit and dispersion responses that contain only few and strong harmonics close to the machine tune. Those eigenvectors are combinations of a large number of small corrector kicks, but their effect on the orbit and dispersion is large because the kicks add up resonantly due to favorable phase relations, as can be seen in Fig. 4. It can be shown that the harmonics of the eigenvectors with the largest eigenvalues always reflect the machine (super) symmetries [13]. Such a spectrum of harmonics is shown for LEP in Fig. 5. For small eigenvalues, the solutions often correspond to orbit and dispersion bumps over some section of the machine.

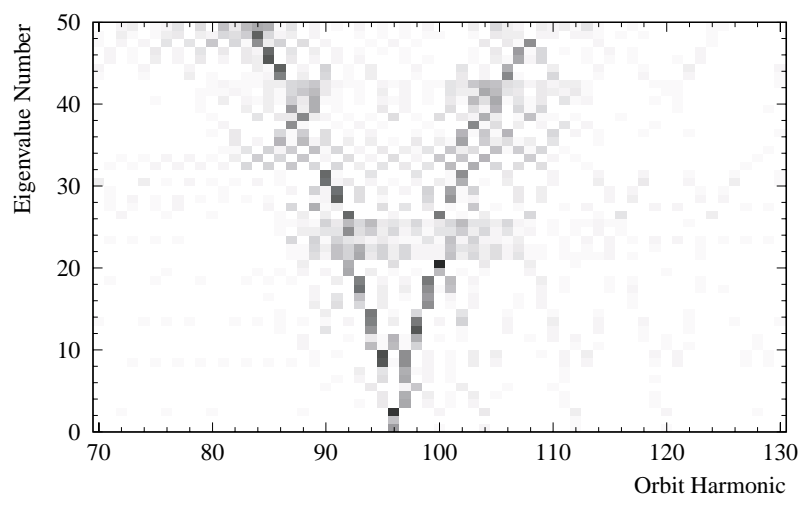

FIG. 5. Harmonics of the normalized vertical orbit eigenvectors $\left(w_{1} \overrightarrow{\boldsymbol{v}}^{(i)} / w_{i}\right)$ for the 50 largest eigenvalues as a function of the eigenvalue number (ordered by decreasing eigenvalue). The strength of the harmonics increases from white to black. The vertical integer tune is 96 . 


\section{B. Orbit corrections with eigenvectors}

Provided that all $w_{i}$ are nonzero, the SVD decomposition can be used to invert matrix $\mathbf{T}$ and solve Eq. (14),

$$
\vec{\theta}=-\mathbf{V W} \mathbf{W}^{-1} \mathbf{U}^{\mathbf{t}} \vec{d}
$$

For $M=N$, Eq. (23) is the solution of the linear system, and, for $M \geq N$, it is the result of the least square minimization. Singular elements, corresponding to values of $w_{i}$ that are very small or zero, can be regularized by setting $1 / w_{i}$ to 0 in matrix $\mathbf{W}^{-1}$.

In practice, the eigenvector decomposition yields a simple way of handling Eq. (23), in particular, when the orbit is corrected with only the $k$ eigenvectors associated to the largest eigenvalues [13]. The measured orbit and dispersion vector $\vec{d}_{m}$ and the corresponding corrector settings $\vec{\theta}_{m}$ are decomposed in terms of orbit and kick eigenvectors

$$
C_{i}=\left(\vec{d}_{m} \cdot \vec{v}^{(i)}\right) \quad \text { and } \quad C_{i}^{c}=\left(\theta_{m} \cdot \vec{\vartheta}^{(i)}\right) .
$$

The vector $\vec{d}_{o}$, defined as

$$
\vec{d}_{o}=\sum_{i=1}^{N} C_{i} \overrightarrow{\boldsymbol{v}}^{(i)}
$$

corresponds to the correctable part of the orbit and dispersion. The residual orbit and dispersion given by $\vec{d}_{m}-\vec{d}_{o}$ cannot be corrected given the correctors that are installed in the machine. This residual is generated by the corrector density, the BPM errors, and the machine alignment. A correction based on the $k$ largest eigenvalues corresponds to the following corrector setting increments:

$$
\vec{\theta}_{c}=\sum_{i=1}^{k}-\frac{C_{i}}{w_{i}} \vec{\vartheta}^{(i)} .
$$

For $k=N$, Eqs. (26) and (23) are identical. For a bare orbit correction, where the effect of already used correctors is first unfolded, the new absolute corrector settings are

$$
\vec{\theta}_{c}=\sum_{i=1}^{k}\left(-\frac{C_{i}}{w_{i}}+C_{i}^{c}\right) \vec{\vartheta}^{(i)}
$$

Figure 6 gives an example of the prediction for orbit, dispersion, and corrector strengths in the case of a vertical bare orbit correction at LEP. Good corrections for the dispersion and the orbit are already obtained with approximately 80 eigenvectors. Since the rms strength of the correctors increases smoothly with the number of eigenvalues, the corrector kicks can be controlled by limiting the number of eigenvectors for the correction.

The SVD algorithm is also suited to identify suspicious monitor readings. Because corrections based only on the largest eigenvalues act principally on the main harmonics of the orbit and the dispersion, local structures and particularly suspicious data become more visible. The simultaneous correction of the dispersion is also a protection against spurious local bumps, since such bumps generate local orbit distortions but global dispersion waves.

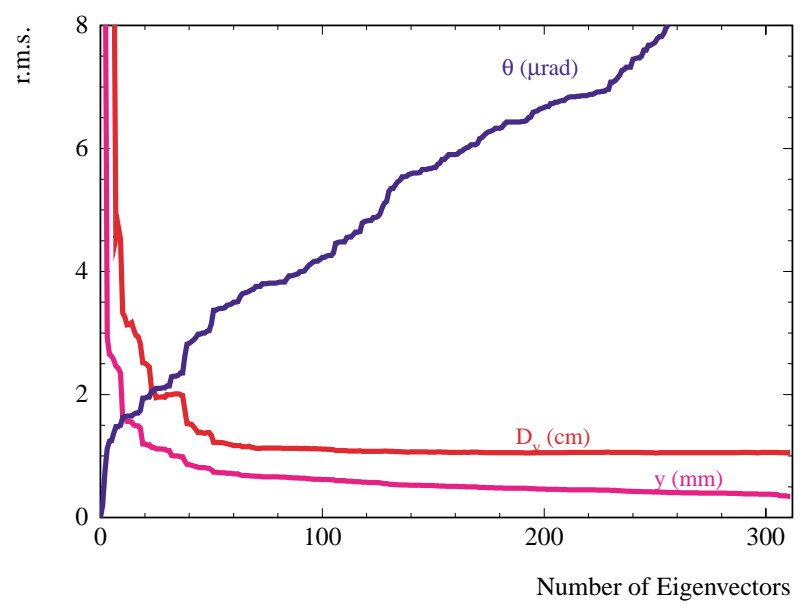

FIG. 6. (Color) Predicted rms of the vertical orbit ( $y$ ), dispersion $\left(D_{y}\right)$, and corrector kicks $(\theta)$ for a bare orbit correction as a function of the number of used eigenvectors (for $\alpha=0.2$ and $\beta=0)$.

\section{Orbit corrections with MICADO}

MICADO [14] is a least square algorithm based on Householder transformations which is widely used for orbit corrections. It is a fast algorithm which performs an iterative search for the most effective corrector. MICADO is, together with SVD, one of the most common orbit correction algorithms. For a nonsingular matrix, a MICADO correction with all $N$ correctors and an SVD correction with all $N$ eigenvectors yield identical solutions. For corrections with a limited number of correctors or eigenvectors, and for singular matrices, the two algorithms behave differently.

A major difference between SVD and MICADO is the corrector strength distribution, MICADO using fewer but also much stronger kicks. The corrector strength rms can be easily controlled with SVD over the number of eigenvalues that are included in a correction; see Fig. 6.

A correction of a small number of localized kicks is handled very effectively by MICADO, particularly when the response matrix is accurate, in which case MICADO can be used to identify the sources of the kicks. On the other hand, corrections based on a few eigenvectors with the largest eigenvalues are similar to corrections of the main harmonics. Such a scheme spreads out the correction of a few kicks over the whole machine which can be an asset when the strength of correctors is limited. To compensate an isolated kick locally, a large number of eigenvectors must be included in the correction such that the linear combination forming $\vec{\theta}_{c}$ yields a single nonzero corrector.

To correct a large number of small imperfections and misalignments, for example, in the case of a well aligned machine, the SVD algorithm may provide a more natural solution by distributing a large number of small kicks over the machine. The concentration of the correction onto a limited number of correctors by MICADO is very efficient but may not always be natural. 
Singularities of the response matrix, associated to very small eigenvalues, are handled more easily with SVD, since it is sufficient to avoid using the corresponding eigenvectors in the corrections procedure. There is no need to disable correctors to regularize the response matrix. A nonzero kick strength weight $\beta$ can also be used to avoid disabling correctors with MICADO.

\section{EXPERIMENTAL RESULTS OF DISPERSION FREE STEERING AT LEP}

Dispersion free steering was tested in 1998 and implemented in the LEP control system for the 1999 run. The response matrices are evaluated from the machine model with the MAD program [15]. The large energy loss per turn at high energy $(\approx 2 \%)$, which affects the response matrices of the two beams differently, is taken into account. The machine model is accurate enough to converge extremely well, even when all corrector settings are unfolded in the case of bare corrections. Corrections can be evaluated for the individual beams, for both beams at the same time, or for the average of the two beams (the most frequent case). Since the size of matrix $\mathbf{T}$ can be as large as $\approx 2300 \times 300$, the SVD algorithm is not applied directly to matrix $\mathbf{T}$ but to the symmetric $N \times N$ matrix $\mathbf{T}^{\mathbf{t}} \mathbf{T}$ of Eq. (19). The eigenvalue problem of Eq. (19) can be solved by a variety of other mathematical algorithms other than SVD; see Ref. [11]. A comparison of different algorithms confirmed the numerical stability of the results. The optimum value for $\alpha$ ranges between 0.1 and 0.3 and, in general, $\alpha$ is set to 0.2 (for an orbit rms expressed in $\mathrm{mm}$ and a dispersion rms expressed in $\mathrm{cm}$ ). This value is in agreement with estimates based on the machine alignment and the accuracy of the dispersion measurement. Results are not very sensitive to the precise value of $\alpha$. $\beta$ is usually set to 0.1 to avoid problems with singular solutions localized in the low-beta insertions.

\section{A. Dispersion correction with single beams}

A small vertical dispersion is obtained with DFS using base line corrections on bare orbit and dispersion in both planes. Only 80 to 120 eigenvectors are used to limit the rms kick strengths to $\approx 5-6 \mu \mathrm{rad}$. This number matches the typical quadrupole kick of approximately $6 \mu \mathrm{rad}$ due to the vertical rms quadrupole misalignment of $150 \mu \mathrm{m}$, although only 312 out of over 800 quadrupoles are equipped with a nearby vertical orbit corrector. Increasing the number of eigenvalues does not, in general, improve the performance. DFS corrections based on the MICADO algorithm yield a good rms dispersion and orbit but larger emittances and worse luminosity performance. This observation may indicate that, by using a large number of weak correctors, the SVD algorithm compensates the closed orbit kicks more locally than MICADO, which concentrates the correction over fewer correctors.
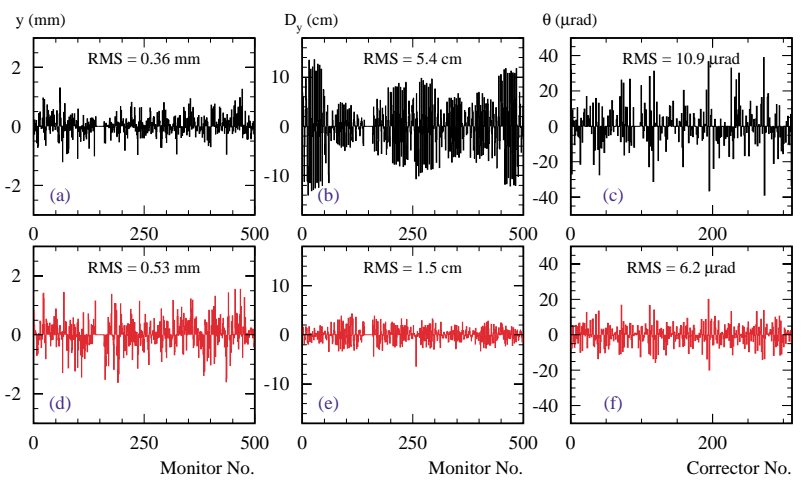

FIG. 7. (Color) The vertical orbit $(y)$, dispersion $\left(D_{y}\right)$, and corrector kick strengths $(\theta)$ after a traditional bare correction of the LEP orbit using MICADO (i.e., $\alpha=0$ ) are shown on the three top figures (a), (b), and (c). The same quantities are shown after a correction with the DFS procedure on the bottom figures (d), (e), and (f). This experiment was performed with a single beam.

A traditional bare orbit correction using MICADO $(\alpha=0)$ is compared to a DFS correction with SVD in Fig. 7. While the orbit rms is not affected significantly, the rms vertical dispersion is reduced from typically 5 to $1.0-1.5 \mathrm{~cm}$, which corresponds to the smallest achievable residual rms dispersion at LEP. For the available momentum range of $\Delta p / p \simeq 0.15 \%$, a dispersion of $1 \mathrm{~cm}$ corresponds to a measured beam position shift of only $15 \mu \mathrm{m}$, at the limit of the LEP BPM resolution. The rms kick strength is reduced by almost a factor of 2 .

Once a good orbit is established, the corrector settings are reused in subsequent runs and the orbit is corrected towards the reference using a few correctors with the MICADO algorithm. The dispersion is stable over time. Small drifts with respect to the optimum are easily corrected using DFS with a MICADO correction.

\section{B. Dispersion correction with two beams}

When LEP is operated above $80 \mathrm{GeV}$, each beam consists of four equidistant bunches. There are eight beam encounters along the circumference, but experiments are installed around only four of the eight collision points. At the four other interaction points the beams are locally separated in the vertical plane. The separation bumps are made with electrostatic elements and produce vertical dispersion of opposite sign for the two beams, which can be reduced only through the design of the separation bumps and the local optics. For the 1999 LEP run the local optics was modified to reduce the rms dispersion generated by those bumps from 2.5 to $1.5 \mathrm{~cm}$. This guarantees that their contribution to the vertical emittance is negligible. The smallest rms dispersion of $1.5 \mathrm{~cm}$ obtained during LEP operation in 1999 corresponds to the minimum originating from the separation bumps. The separation bumps and the resulting dispersion difference between the beams are shown in Fig. 8. 

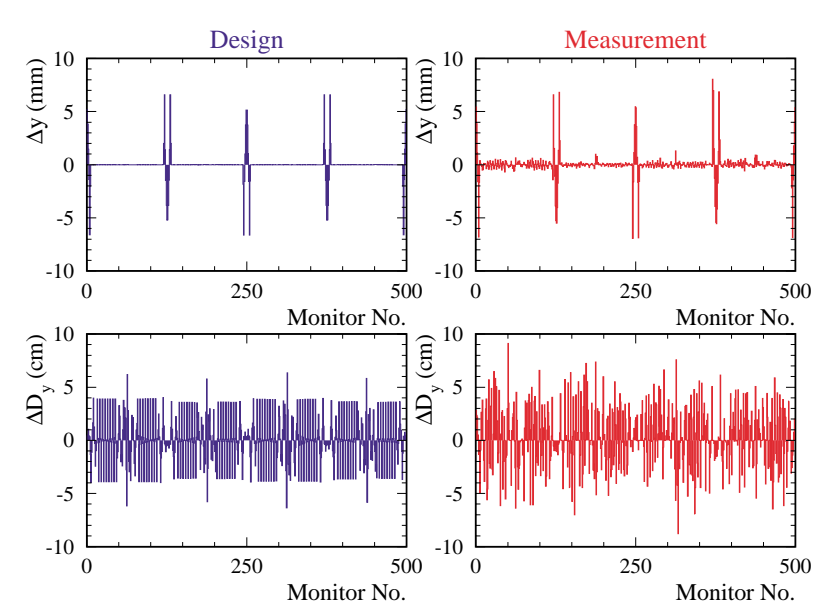

FIG. 8. (Color) Design (left) and measured (right) vertical orbit difference $\Delta y$ and dispersion difference $\Delta D_{y}$ between the $e^{+}$ and $e^{-}$beam. The four separation bumps are clearly visible on the closed orbit difference. The measured rms orbit difference outside the separation bumps is $0.2 \mathrm{~mm}$. The residual vertical dispersion difference of $3.1 \mathrm{~cm}$ is generated by the separation bumps. This corresponds to a dispersion of $1.5 \mathrm{~cm}$ for each beam, but of opposite sign for $e^{+}$and $e^{-}$.

\section{Performance improvements}

The vertical emittance extracted from the measured luminosity for 1998 and 1999 is shown in Fig. 9. At LEP the strong beam-beam effect, with values of the beam-beam parameter exceeding 0.08 , blows up the vertical beam size and prevents a linear increase of the beam-beam parameter with bunch current. To avoid strong biases due to beam-beam blowup, the emittances are compared for a similar range of bunch currents. The vertical emittance is extracted from the measured luminosity using the design vertical betatron function at the collision point and the design horizontal emittance. Measurements of the horizontal beam spots at the collision points using beam-beam deflection scans [16] indicate that the blowup in the horizontal plane is small at the currents that are considered here.

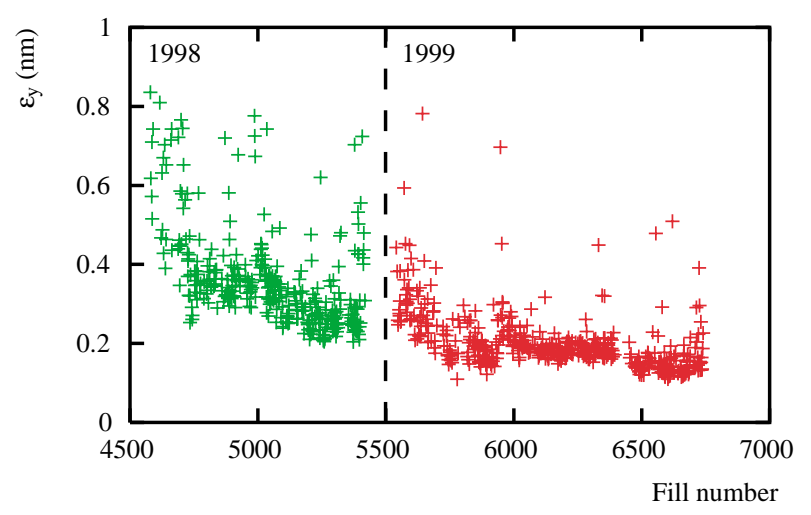

FIG. 9. (Color) Average vertical beam emittance in collision for bunch currents of 500 to $550 \mu \mathrm{A}$ for each LEP fill in 1998 and 1999. The emittance is extracted from the measured luminosity.

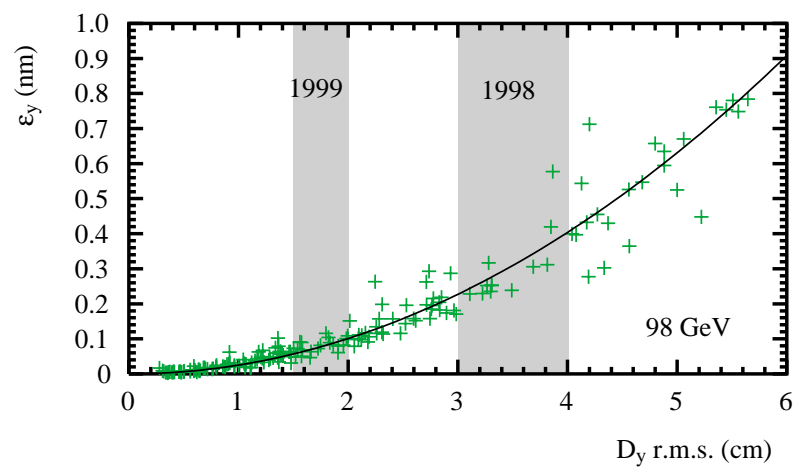

FIG. 10. (Color) Simulated dependence of the vertical emittance $\varepsilon_{y}$ on the rms vertical dispersion. The typical vertical dispersion rms for 1998 and 1999 are indicated by the two bands.

From Fig. 9 it is clear that with the help of DFS the vertical emittance in 1999 was rapidly pushed below the best values of the 1998 run. Contrary to previous years where the search for good (golden) orbits was done empirically, in 1999 the base line performance was established deterministically with DFS. The best emittance values were reduced by almost a factor of 2 . They correspond to emittance ratios of $0.6 \%\left(\varepsilon_{x} \simeq 25 \mathrm{~nm}\right)$. This ratio is often quoted as an indicator for the beam quality, but it is important to note that the concept of emittance ratio is not adequate for our case since $\varepsilon_{y}$ is not dominated by coupling. At LEP the emittance ratio can be changed artificially by varying $\varepsilon_{x}$, and ratios below $0.5 \%$ can be obtained for larger $\varepsilon_{x}$.

The dependence of the vertical emittance $\varepsilon_{y}$ on the rms vertical dispersion was simulated with the MAD program and is shown in Fig. 10. $\varepsilon_{y}$ scales with the square of the rms vertical dispersion [see Eq. (3)], albeit with a spread that depends on the details of the orbit and dispersion pattern. The simulated and measured emittances agree quite well. Since the rms dispersion was reduced from 3-4 cm in 1998 down to $1.5-2 \mathrm{~cm}$ in 1999 with DFS, an emittance improvement by a factor of 4 would be expected, while only a factor of 2 was observed. The difference may be explained by a larger beam-beam blowup for smaller $\varepsilon_{y}$ [17]. It is also possible that the beam sizes at the interaction points were limited by local coupling between the two planes, in which case the determination of the emittance from the luminosity overestimates the true value. Attempts to improve the performance by tuning the local coupling did not yield important gains.

\section{CONCLUSION}

Dispersion free steering, a deterministic and simultaneous correction of the closed orbit and the dispersion, was implemented in LEP. The correction scheme is relying mainly on the SVD algorithm to solve the least square problem. The vertical dispersion in LEP was reduced to the expected minimum, only limited by residual dispersion 
generated from separation bumps and by the measurement noise. With DFS the empirical search for golden orbits yielding peak performance could be made deterministic and a significantly smaller residual dispersion was obtained. This resulted in a vertical emittance gain of approximately $50 \%$.

[1] G. Guignard, in Proceedings of the Fifth Advanced Accelerator Course, edited by S. Turner (CERN, Geneva, 1995), pp. $43-76$.

[2] D. Brandt, H. Burkhardt, M. Lamont, S. Myers, and J. Wenninger, Rep. Prog. Phys. 63, 939-1000 (2000).

[3] R. Bailey et al., in Proceedings of the 1995 Particle Accelerator Conference, Dallas, Texas (IEEE, New York, 1995), pp. 548-550.

[4] R. Assmann and K. Cornelis, CERN Report No. CERNSL-2000-046-OP, 2000.

[5] M. Lamont, in Proceedings of the 8th Workshop on LEP Performance, Chamonix, edited by J. Poole (CERN, Geneva, 1998), pp. 134-137.

[6] T. Raubenheimer and R. Ruth, Nucl. Instrum. Methods Phys. Res., Sect. A 302, 191 (1991).

[7] R. Assmann, T. Chen, F. J. Decker, M. Minty, T. Raubenheimer, and R. Siemann, in Proceedings of the International Workshop on Accelerator Alignment, Tsukuba (KEK, Tsukuba, 1996), pp. 463-477.
[8] R. Assmann et al., SLAC Report No. SLAC-PUB-7782, 1998.

[9] M. Donald, Y. Cai, H. Shoaee, and G. White, in Proceedings of the 17th Particle Accelerator Conference, Vancouver, edited by M. Comyn et al. (IEEE, New York, 1997), pp. 1454-1457.

[10] J. Safranek, in Handbook of Accelerator Physics and Engineering, edited by A. Chao and M. Tigner (World Scientific, Singapore, 1999), pp. 273-276.

[11] W. Press, B. Flannery, S. Teukolsky, and W. Vetterling, Numerical Recipes (Cambridge University, Cambridge, England, 1987), 1st ed.

[12] S. Krinsky, in Handbook of Accelerator Physics and Engineering (Ref. [10]), pp. 267-268.

[13] E. Bozoki and A. Friedman, Nucl. Instrum. Methods Phys. Res., Sect. A 344, 269 (1994).

[14] B. Autin and Y. Marti, CERN Report No. ISR MA/73-17, 1973.

[15] H. Grote and C. Iselin, CERN Report No. CERN-SL/ 90-13 Rev. 3 (AP), 1990.

[16] C. Bovet, M. Hildreth, M. Lamont, H. Schmickler, and J. Wenninger, in Proceedings of the 5th European Particle Accelerator Conference, Sitges, Spain, edited by S. Myers et al. (IOP, Bristol, 1996), pp. 325-327.

[17] R. Assmann, in Proceedings of the Xth Workshop on LEP-SPS Performance, Chamonix, edited by J. Poole (CERN, Geneva, 2000), pp. 259-268. 